\title{
Topical Antibiotics Reduce CD11c+ Cell Numbers in the Healthy Murine Cornea and Modulate their Response to Contact Lens Wear
}

\author{
Ananya Datta \\ University of California, Berkeley \\ Justin Lee \\ University of California, Berkeley \\ Tiffany Truong \\ University of California, Berkeley \\ David J. Evans
}

Touro University California

Suzanne M. J. Fleiszig ( $\nabla$ fleiszig@berkeley.edu )

University of California, Berkeley

\section{Research Article}

Keywords:

Posted Date: February 25th, 2022

DOI: https://doi.org/10.21203/rs.3.rs-1304551/v1

License: (c) (i) This work is licensed under a Creative Commons Attribution 4.0 International License. Read Full License 


\section{Abstract}

Previously we reported contact lens-induced CD11c + cell responses in healthy mouse corneas, a phenomenon that also occurs in humans. To test involvement of ocular-associated bacteria, we examined the impact of topical antibiotics on corneal CD11c + cell populations during $24 \mathrm{~h}$ of lens wear. Corneas were treated with gentamicin and ofloxacin $(0.3 \%)$ or gentamicin alone, some also treated prior to lens wear (24 h). Contralateral PBS-treated eyes served as controls. CD11c-YFP mice allowed CD11c + cell visualization. Viable ocular- or lens-associated bacteria were labeled using FISH (16S rRNA-targeted probe) or click-chemistry (alkDala). Antibiotic treatment reduced baseline CD11c + cell numbers without lens wear and suppressed CD11c + cell responses to lens wear if corneas were both pretreated and treated during wear. Few bacteria colonized corneas or lenses under any circumstances. Conjunctival commensals were significantly reduced by antibiotics with or without lens wear, but minimally impacted by lens wear alone. Deliberate inoculation with conjunctival commensals triggered CD11c + cell responses irrespective of antibiotic pretreatment. These results suggest that while lens wear does not necessarily increase quantifiable numbers of conjunctival commensals, those neutralized by antibiotics play a role in lens-associated CD11c + cell responses and maintaining baseline CD11c + cell populations

\section{Introduction}

The healthy cornea of the eye is remarkably resistant to bacterial infection but can be rendered susceptible by injury or contact lens wear. Research by us and others has shown that this resistance depends on a constitutive defensive combination of tear fluid, epithelial and basal lamina barrier function, and the ability of resident immune cells to detect and respond to injury, microbes or their ligands ${ }^{1}$. In this regard, the cornea contains a small resident population of bone-marrow derived dendritic cells and macrophages in the stroma and epithelium ${ }^{2,3}$, with centrally-located stromal dendritic cells being primarily immature precursors that can mature and acquire MHC Class II expression during inflammatory events ${ }^{2,3}$.

The mechanisms by which contact lens wear predisposes to corneal infection by $P$. aeruginosa and other bacteria are yet to be resolved. Recently, we reported the development of a murine model of contact lensassociated $P$. aeruginosa keratitis in which lens wear was associated with a corneal para-inflammatory response involving CD11 $\mathrm{c}+$ (dendritic cell) infiltration/activation by $24 \mathrm{~h}$ and $\mathrm{Ly} 6 \mathrm{G}+\mathrm{cell}$ (neutrophil) infiltration after 5-6 days ${ }^{4}$, despite corneas remaining free of visible pathology. Importantly, contact lensassociated corneal para-inflammatory responses involving Langerhans cells have also been reported in humans ${ }^{5,6}$.

Previously, we showed that $\mathrm{CD} 11 \mathrm{c}+$ cells in murine corneas respond to $P$. aeruginosa challenge in vivo, contributing to IL-1R-dependent epithelial barrier function against bacterial adhesion after superficial injury ${ }^{7}$. Here, we sought to determine the role of bacteria in triggering corneal CD11c + responses to contact lens wear. While healthy corneas do not host resident viable bacteria ${ }^{8}$, adjacent tissues are colonized with a microbiome. For example, the conjunctiva hosts numerous resident bacteria including 
Staphylococcus spp, and Corynebacterium spp. ${ }^{9,10}$, the latter shown to be important for corneal immune responses during active infectious disease ${ }^{11}$. Contact lens wear can potentially alter bacterial colonization in the conjunctiva ${ }^{12}$, and cornea ${ }^{13}$, including via lens binding ${ }^{14,15}$. Indeed. in the murine lens-wear model, and in humans, worn lenses were found colonized by bacteria typical of those found on the skin and conjunctiva ${ }^{4,14,16}$. To explore the involvement of bacteria in corneal CD11c + responses to lens wear, CD11c+-YFP mice were treated with topical antibiotics with and without contact lens wear, and imaging used to quantify $\mathrm{CD} 11 \mathrm{c}+$ responses in addition to conjunctival and lens colonization by viable bacteria. Results showed that topical antibiotic treatment can reduce resident CD11c + cell numbers and suppress their responses to contact lens wear. They also showed that corneal CD11c + cell responses could be induced in healthy corneas using conjunctival commensals. Taken together, these results suggest the involvement of commensal bacteria in driving the CD11c + cell responses.

\section{Results}

Topical antibiotic application decreases $\mathrm{CD} 11 \mathrm{c}+$ cell numbers in the healthy murine cornea. Prior to exploring topical antibiotic effects on contact lens-associated CD11c + responses, a control experiment was performed to determine if antibiotics affected baseline numbers of resident corneal CD11c + cells. CD11c-YFP mice were treated topically with a cocktail of gentamicin and ofloxacin $(0.3 \%$ of each in solution), or gentamicin alone, from $0-6 \mathrm{~h}$ and corneal $\mathrm{CD} 11 \mathrm{c}+\mathrm{cell}$ numbers imaged and quantified at $24 \mathrm{~h}$ (Methods Procedure 1). Figure 1 shows that both antibiotic treatments caused a reduction of corneal CD11c + cell numbers versus PBS-treated contralateral controls. PBS ( $44 \pm 4$ cells) versus antibiotic cocktail ( $28 \pm 3$ cells, $6 \%$ decrease) or gentamicin ( $33 \pm 2$ cells, $25 \%$ decrease) $(P<0.0001$ One-Way ANOVA Tukey's multiple comparisons test, Fig. $1 \mathrm{a} \& \mathrm{~b}$ ). There was no significant difference between antibiotic cocktail and gentamicin alone. FISH labeling confirmed an absence of viable bacteria on antibiotic-treated corneas as for healthy murine corneas (Supplemental Fig. S1).

\section{Topical gentamicin application modulates contact lens-associated CD11c + cell responses. Having} shown that gentamicin alone was not significantly different from its combination with ofloxacin, here we used gentamicin to explore the role of bacteria in contact lens-associated CD11c + cell responses (increased number and changes in morphology) in the murine cornea after $24 \mathrm{~h}$ of lens wear ${ }^{4}$. We tested if topical gentamicin treatment could abrogate that response. Mice were divided into PBS or gentamicin treated groups and fitted with a contact lens on one eye for $24 \mathrm{~h}$ (Methods Procedure 2). Contralateral eyes served as no lens wear controls. Figure 2 shows that $24 \mathrm{~h}$ of contact lens wear was associated with a significant increase in CD11c + cell numbers in both the PBS control group ( $\sim 44 \%$ increase) and the gentamicin-treated group ( 36\% increase) (Fig. 2a \& b). However, no significant change in CD11c + cell morphology (circularity) was observed under any condition (Fig. 2c). Thus, despite a reduction in baseline $\mathrm{CD} 11 \mathrm{c}+$ cells with gentamicin treatment, contact lens-induced CD11c + cell responses after $24 \mathrm{~h}$ remained intact. 
Since potential antibiotic effects may require extended exposure time, the experiment shown in Fig. 2 was repeated but eyes were first pretreated with either PBS or gentamicin (Methods Procedure 3). Under these conditions, topical gentamicin blocked the $24 \mathrm{~h}$ contact lens-associated corneal CD11 + cell response (Fig. 3a \& b). If only gentamicin pretreatment was applied, the $24 \mathrm{~h}$ contact lens-associated CD11 + cell response remained intact. This experiment also showed that the reduction in corneal CD11c + cells induced by topical gentamicin was maintained for $24 \mathrm{~h}$ after antibiotic removal. As above, no significant differences in CD11C + cell morphology were detected between groups (Fig. 3c).

Antibiotic treatment reduced the number of viable conjunctival bacteria detected by alkDala labeling with and without contact lens wear. In both of the above experiments (Figs. 2 \& 3), FISH or alkDala labeling of bacteria on the cornea revealed very few bacteria under all conditions with no significant differences between PBS or gentamicin treated groups and with or without contact lens wear for $24 \mathrm{~h}$ (Supplemental Fig. S2), a finding consistent with our previous work ${ }^{4,8,17}$. Also consistent with our previous work, FISH labeling of worn lenses revealed bacterial binding to worn lenses but without a difference between groups (Supplemental Fig. S2). Thus, increases in corneal CD11c + cell numbers associated with $24 \mathrm{~h}$ of contact lens wear (Figs. 2 \& 3) and their suppression by antibiotic treatment (Fig. 3) were not associated with any changes in the number of viable bacteria detected by FISH on the cornea or worn lenses.

Thus, the adjacent conjunctiva was examined using the same experimental conditions. AlkDala labeling alone was used given its distinctive (bacteria-specific) labeling of the filamentous conjunctival bacteria in mouse eyes observed in our previous studies 8,17 , and because we previously found FISH to be problematic for the conjunctiva. The filamentous nature of the conjunctival bacterial population required quantifying area of alkDala florescence per field of view rather than individual bacterial cells. As shown in Fig. 4, there was a significant decrease in bacterial fluorescence in gentamicin treated eyes versus PBS controls with and without contact lens wear, showing a reduction in the population detectable. These results suggested a potential relationship between the conjunctival population of commensals and the corneal CD11c + cell responses during gentamicin treatment with and without lens wear (comparing Fig. $4 a$ \& b with Figs. $2 b \& 3 b$, respectively).

\section{Addition of commensal conjunctival bacteria restores $\mathrm{CD} 11 \mathrm{c}+$ cell numbers in antibiotic treated murine}

corneas. Having found that changes in corneal CD11c + cells associated with gentamicin treatment and contact lens wear correlated with the detection of viable conjunctival bacteria using alkDala labeling, we next explored if we could complement the CD11c+ response using conjunctival commensal inoculation. Thus, post-gentamicin treated eyes (healthy corneas) were inoculated in vivo with Gram-positive commensals (mouse-derived C. mastitidis, and human-derived coagulase-negative staphylococci). We also used the opportunistic pathogen $P$. aeruginosa that we previously showed can induce $\mathrm{CD} 11 \mathrm{c}+\mathrm{cell}$ responses in mouse corneas ${ }^{7}$. After 4 h exposure to $\sim 10^{11} \mathrm{CFU} / \mathrm{mL}$ bacteria, corneal CD11c + cell numbers were quantified ex vivo. Fig. 5 shows that the exposure of previously gentamicin-treated eyes to Gram-positive commensal bacteria restored corneal CD11c + numbers to the same level as control nonantibiotic treated eyes after bacterial challenge, i.e. showing that the reduction in CD11c + cells induced by prior antibiotic treatment could be complemented by the addition of conjunctival commensals (Fig. 5). 
While $P$. aeruginosa also increased corneal CD11c + cell numbers, levels in gentamicin treated eyes, remained below those of control untreated eyes suggesting a commensal is needed to complement the CD11c + cell response after antibiotic treatment.

\section{Discussion}

Previously, we reported development of a murine model of contact lens wear in which we demonstrated lens-associated corneal para-inflammation after 5-6 days of wear, that was preceded by infiltration and activation of corneal CD11 c+ (dendritic) cells after $24 \mathrm{~h}^{4}$. The latter response mimicked those observed for Langerhans cells during human lens wear ${ }^{18,19}$. Here, we investigated the potential role of microbial triggers in contact lens-induced CD11c + responses in the murine model. Using topical application of broad-spectrum antibiotics to target ocular-associated bacteria with and without lens wear, results showed that antibiotic exposure reduced baseline CD11c + cell levels in healthy corneas after $24 \mathrm{~h}$ and suppressed corneal CD11 + cell responses to $24 \mathrm{~h}$ of contact lens wear but only if corneas were pretreated with antibiotic and treatment continued during lens wear. In both cases, this correlated with reduced numbers of viable conjunctival-associated bacteria. Adding commensal bacteria to antibiotictreated eyes restored corneal CD11c + cell numbers: not the case with addition of the potential pathogen $P$. aeruginosa. Together these findings suggest a role for ocular-associated commensal flora in maintaining $\mathrm{CD} 11 \mathrm{c}+$ cell homeostasis and in enabling contact lens-associated para-inflammation. They also appear to be important for the induction of the $\mathrm{CD} 11 \mathrm{c}+$ response to $P$. aeruginosa challenge which we previously showed contributes to resisting $P$. aeruginosa colonization if the cornea is superficiallyinjured ${ }^{7}$.

How might topical antibiotics mediate the CD11c + phenotypes observed in this study? While a combination of ofloxacin and gentamicin was used initially, gentamicin alone was found effective. This is an aminoglycoside antibiotic, with bactericidal activity against many Gram-negative bacteria and some medically-important Gram-positive bacteria ${ }^{20}$. Since there are very few viable bacteria on the murine cornea $^{8}$ (confirmed in the present study), or on contact lenses, the most likely mechanism is that the antibiotic interfered with conjunctival commensals, either directly reducing their viability and/or interfering with their growth or metabolism. Indeed, there was a significant decrease in conjunctival alkDala (peptidoglycan cell wall) labeling after gentamicin treatment closely correlating with changes in baseline levels of CD11c + cells and their responses to contact lens wear.

Involvement of conjunctival-associated commensals in the observed CD11c + phenotypes would not be surprising as others have shown conjunctival-associated bacteria (C. mastitidis) to be important for corneal immune responses against $P$. aeruginosa infection via effects on gamma-delta $T$ cells ${ }^{21}$. However, the CD11c + cell response as a result of lens wear, a completely different phenotype occurring in healthy rather than diseased corneas, could involve different mechanisms. Indeed, other possibilities include that topical gentamicin affects commensal bacteria beyond the eye. The nasolacrimal drainage system exits the eye and drains tear fluid into the back of the nose and throat so topically added 
gentamicin solution at this very high concentration $(3 \mathrm{mg} / \mathrm{ml})$ could influence microbiota of the nasopharynx or intestinal tract, the latter well known to influence immune responses at distant mucosal sites including the conjunctiva ${ }^{22-24}$. Another potential explanation is that topical gentamicin influenced corneal CD11c + cell numbers and responses to lens wear independently of effects on bacteria. Indeed, aminoglycosides and other antibiotics are known to have a multitude of direct impacts on immune cell function ${ }^{25-28}$. Further studies will be needed to delineate the respective roles of bacteria, conjunctival or other, versus other potential mechanisms, in mediating the CD11c + phenotypes observed.

Even if topical ocular gentamicin does mediate the CD11c + phenotypes observed in our study via an impact on microbes, it cannot be assumed that bactericidal effects are exclusively involved. In this study, the presence of numerous filamentous forms of murine conjunctival bacteria ${ }^{8}$ necessitated the quantification of total alkDala (peptidoglycan cell wall) fluorescence rather than distinguishing individual bacterial cells. Thus, it remains possible that alkDala fluorescence with gentamicin exposure resulted from reduced cell wall synthesis while bacteria remained viable. Another possibility is that gentamicin inhibited bacterial protein synthesis (part of its established mechanism of action ${ }^{20}$ ) interfering with the expression of bacterial ligands, metabolites, or other secreted factors that mediate the CD11c+ responses. In this regard, the rapid return of $C D 11 c+$ responses to contact lens wear after gentamicin removal (Fig. 3b) is interesting and may reflect a rapid resumption of bacterial ligand/metabolite/secreted factor expression by conjunctival commensals. It is also interesting that baseline levels of CD11c + cell numbers did not recover in the same time frame after gentamicin removal (Fig. 3b) suggesting the possibility that distinct mechanisms mediate these CD11c + cell phenotypes, or that responsible bacterial factors were trapped under or bound to the contact lens that enabled a response. Related to that possibility, contact lens-associated CD11c + responses (Figs. 2b \& 3b) occurred with little or no observable increase in alkDala fluorescence of conjunctival commensals (Fig. 4a \& b) suggesting that commensal bacteria involvement might not be via direct triggering of contact lensassociated CD11c + cell responses. In other words, some other trigger associated with contact lens wear might instead stimulate already existing conjunctival commensals to drive lens-associated CD11c + responses independently of peptidoglycan cell wall synthesis, e.g. expression of ligands, metabolites etc. Given how dramatically a contact lens can alter the ocular surface environment, conjunctival (or other) commensals may play an even more complex role being only one part of a system regulating responsiveness and homeostasis of corneal CD11c + cells. Our results showing that gentamicin exposure reduced corneal CD11C + responses to P. aeruginosa but not to known commensals (Fig. 5) would be consistent with a more complex mechanism.

In summary, the results of this study show that topical antibiotic application modulates corneal CD11c+ cells influencing their constitutive (baseline) levels and ability to respond to contact lens wear or $P$. aeruginosa challenge. While the results implicate commensal conjunctival bacteria in these CD11c+ phenotypes, further studies will be needed to determine the nature of their contribution which the data suggest might be complex. 


\section{Methods}

Mice. Transgenic wild-type C57BL/ 6 female mice (6-8 weeks old) were used that express both a CD11CYFP reporter (CD11c-positive cells, e.g. dendritic cells, yellow) and $m T / m G$ knock in (cell membranes, red) 4,7 . At the end of each experiment, or if mice presented with excessive weight loss, distress, signs of keratitis, euthanasia was performed using isoflurane inhalation (5\%) for $10 \mathrm{~min}$, or combined administration (intra-peritoneal injection) of ketamine $(80-100 \mathrm{mg} / \mathrm{Kg}$ ) and xylazine $(5-10 \mathrm{mg} / \mathrm{Kg}$ ), either method followed by cervical dislocation. Eyes were enucleated for fixation and imaging. All procedures involving animals were carried out in accordance with the standards established by the Association for the Research in Vision and Ophthalmology, under a protocol AUP-2019-06-12322 approved by the Animal Care and Use Committee, University of California Berkeley. This protocol adheres to PHS policy on the humane care and use of laboratory animals, and the guide for the care and use of laboratory animals. This study is reported in accordance with the ARRIVE guidelines (Animals in Research: Reporting In-Vivo Experiments).

Topical antibiotic treatment. One or both eyes of each mouse were treated topically with a $5 \mu$ ldrop of antibiotic solution: a combination (cocktail) of gentamicin and ofloxacin (Falcon Pharmaceuticals. Fort Worth, TX USA) in solution (0. \% of each), or gentamicin alone in solution (0. \%) for 3 applications during time $0-6 \mathrm{~h}$. Control eyes were treated with PBS. Eyes were examined for various phenotypes at $24 \mathrm{~h}$. In some experiments, eyes were pretreated with antibiotic solution or PBS using the same timing prior to the above treatment.

Contact lens fitting. In some experiments, mice were fitted with custom-manufactured silicone hydrogel contact lens as previously described ${ }^{4}$. Contact lenses were fitted only onto the right eye and the contralateral eye used as a non-lens wearing control. Before fitting, contact lenses were removed from their packaging solution and soaked for $1 \mathrm{~h}$ in sterile phosphate buffered saline (PBS). After lens insertion under isoflurane anesthesia, mice were single-housed without enrichment to prevent lens removal. Pure-o'Cel paper bedding (The Andersons Inc., Maumee $\mathrm{OH}$ ) was used to reduce dust level. For overnight lens wear mice were returned to the animal care facility. Mice were monitored daily for lens retention, and evidence of pathology, e.g. discharge or corneal opacity. Any mouse that lost its contact lens was excluded from further experimentation.

Fluorescein in-situ hybridization (FISH). FISH-labeling was performed as previously described using universal 16S rRNA-targeted gene [Alexa488]-GCTGCCTCCCGTAGGAGT-[Alexa488] (Eurofins Genomics) ${ }^{8}$. Contact lenses or enucleated eyes were fixed in paraformaldehyde $(2 \%)$ for $24 \mathrm{~h}$ then stored in PBS at $4^{\circ} \mathrm{C}$ until processed for FISH. Samples were washed in $80 \% \mathrm{EtOH}, 95 \% \mathrm{EtOH}$ then PBS for 10 min each with rotation, placed in hybridization buffer [ $\mathrm{NaCl}(0.9 \mathrm{M})$, Tris- $\mathrm{HCl}(20 \mathrm{mM}, \mathrm{pH} 7.2)$ and SDS (0.01\%)] for incubation $\left(55^{\circ} \mathrm{C}, 30 \mathrm{~min}\right)$, then incubated overnight at $55^{\circ} \mathrm{C}$ with the $16 \mathrm{~S}$ rRNA probe $(100 \mathrm{nM})$. Samples were transferred to wash buffer solution $[\mathrm{NaCl}(0.9 \mathrm{M})$ and Tris $-\mathrm{HCl}(20 \mathrm{mM}, \mathrm{PH} 7.2)]$, then washed three times for 10 min each with rotation. For imaging, contact lenses were flat-mounted with Prolong Gold ${ }^{\text {TM }}$ 
and eyes were whole-mounted in a $47 \mathrm{~mm}$ Petri dish filled with PBS to cover the eyeball completely. Bacteria were imaged and quantified using ImageJ with maximum intensity projections.

AlkDala (Alkyne-Functionalized D-Alanine) Labeling. Viable bacteria on the murine ocular surface were also labeled using an alkyne functionalized D-alanine (alkDala) biorthogonal probe as previously described ${ }^{8,29,30}$. Freshly enucleated eyes were incubated in a solution of alkDala (10 mM) in Dulbecco's Modified Eagle Medium (DMEM) at 37. C for $2 \mathrm{~h}$ then transferred to pre-chilled 70\% EtOH then fixed for 20 min at $-20 \circ \mathrm{C}$. After a thorough rinse in PBS, eyes were permeabilized in PBS with Triton-X100 (0.5\%) for 10 min with shaking at room temperature and washed 3 times for 5 min each in PBS with Triton-X100 $(0.1 \%)$ and BSA $(3 \%)$ with shaking. Eyes were transferred to click-labeling cocktail [in PBS, TBTA (100 $\mu \mathrm{M}), \mathrm{CuSO}_{4}(1 \mathrm{mM})$, sodium ascorbate $(2 \mathrm{mM})$, a $488 \mathrm{~nm}$ azide-fluorophore $(10 \mu \mathrm{M})$, and BSA (0.1 $\mathrm{mg} / \mathrm{mL}$ )] for $1 \mathrm{~h}$ with shaking at room temperature. Eyes were imaged as described below.

Imaging. Confocal imaging of the mouse cornea (whole eyes ex vivo), or worn contact lenses, was performed using a 20x/1.00 NA water-dipping objective with an upright Olympus Fluoview FV1000 microscope. Whole mounted eyes were imaged using a $512 \mathrm{~nm}$ laser for detection of CD11c+-YFP cells (Yellow Fluorescence Protein) and a $559 \mathrm{~nm}$ laser for detecting red fluorescent cell membranes. A $488 \mathrm{~nm}$ laser was used to detect bacteria labeled with FISH or alkDala. For Z stacks at $1.00 \mu \mathrm{m}$ steps, images were collected from 4 or more random fields per sample. 3-D image reconstruction (reducing a 3-D image into 2-D) was achieved by projecting the maximum intensity of each pixel in a specific channel to the $z$ plane. Image-J (MorpholibJ tools collection) and Imaris (Bitplane) were used for image processing and analysis. For circularity measurements, morphological image analysis was performed using 3D segmentation in ImageJ and parameters related to z-projections used (circularity) to exclude artifacts due to lower $\mathrm{z}$ resolution.

Bacteria. Three bacterial species were used in this study. Corynebacterium mastiditis ${ }^{11}$ (kindly provided by Dr. Anthony St. Leger, University of Pittsburgh, PA), a clinical isolate of coagulase-negative Staphylococcus spp. and Pseudomonas aeruginosa strain PA01F. Bacteria were grown on tryptic soy agar plates at $37^{\circ} \mathrm{C}$ for $\sim 16 \mathrm{~h}$. A bacterial inoculum was prepared by suspension in PBS to a concentration of $\sim 10^{11} \mathrm{CFU} / \mathrm{ml}$ that was confirmed by viable counts. To inoculate mouse eyes, mice were anesthetized using ketamine $(80-100 \mathrm{mg} / \mathrm{Kg})$ and dexmedetomidine $(0.25-0.5 \mathrm{mg} / \mathrm{Kg})$ and the cornea of one eye was inoculated with $5 \mu \mathrm{l}$ of bacterial suspension. An additional $5 \mu \mathrm{l}$ of bacterial suspension was re-inoculated onto the corneas each hour up to $4 \mathrm{~h}$ ( 4 inoculations per eye in total). Mice remained anesthetized and covered under a heat lamp for the entire $4 \mathrm{~h}$ period. Subsequently, mice were subject to euthanasia and eyes examined by quantitative imaging.

\section{Experimental procedures.}

Procedure 1. Mice were divided into experimental groups containing 3-4 mice. One group was treated with antibiotic solution (cocktail), another with gentamicin alone, in one eye as described above. 
Contralateral eyes were treated with PBS as a control. After $24 \mathrm{~h}$, eyes were imaged ex vivo to measure corneal CD11c + cell numbers or subject to FISH labeling to determine if viable bacteria were present.

Procedure 2. Two groups of 3-4 mice were used. One group was treated with gentamicin solution (timing as above) in both eyes, the other group similarly treated in both eyes with PBS as a control. For the antibiotic treated group, after the first application of antibiotic, contact lens fitting was performed on one eye followed by remaining antibiotic applications. Contralateral eyes served as non-lens wearing antibiotic-treated controls. The PBS control group was treated similarly. One eye fitted with a contact lens followed by remaining PBS applications, contralateral eyes served as non-lens wearing PBS-treated controls.

Procedure 3. Similar to Procedure 2 with two groups of 3-4 mice: antibiotic treated group versus PBS control group. However, prior to each experiment, each group was pretreated with either antibiotic or PBS with the same timing as used for the primary experiment. In some experiments, however, antibiotic pretreatment was followed by contact lens application with PBS or PBS alone.

Statistical analysis. Data analysis was performed using Prism 9.0 for Mac, Microsoft Excel 2010, and the Statistical Package for Social Science for Mac version 27.0 (SPSS, Inc, Chicago, IL). The distribution of data was assessed by the normality test (Shapiro-Wilk test and Kolmogorov-Smirnov test), and since most data was normally distributed, it was expressed as the mean with standard deviation. One-way or Two-way ANOVA with Tukey's multiple comparisons test for post-hoc analysis. P values less than 0.05 were considered significant.

Mice. Transgenic wild-type C57BL/ 6 female mice (6-8 weeks old) were used that express both a CD11CYFP reporter (CD11c-positive cells, e.g. dendritic cells, yellow) and $m T / m G$ knock in (cell membranes, red) 4,7 . At the end of each experiment, or if mice presented with excessive weight loss, distress, signs of keratitis, euthanasia was performed using isoflurane inhalation (5\%) for $10 \mathrm{~min}$, or combined administration (intra-peritoneal injection) of ketamine $(80-100 \mathrm{mg} / \mathrm{Kg})$ and xylazine $(5-10 \mathrm{mg} / \mathrm{Kg})$, either method followed by cervical dislocation. Eyes were enucleated for fixation and imaging. All procedures involving animals were carried out in accordance with the standards established by the Association for the Research in Vision and Ophthalmology, under a protocol AUP-2019-06-12322 approved by the Animal Care and Use Committee, University of California Berkeley. This protocol adheres to PHS policy on the humane care and use of laboratory animals, and the guide for the care and use of laboratory animals. This study is reported in accordance with the ARRIVE guidelines (Animals in Research: Reporting In-Vivo Experiments).

Topical antibiotic treatment. One or both eyes of each mouse were treated topically with a $5 \mu$ ldrop of antibiotic solution: a combination (cocktail) of gentamicin and ofloxacin (Falcon Pharmaceuticals. Fort Worth, TX USA) in solution (0. \% of each), or gentamicin alone in solution (0. \%) for 3 applications during time $0-6 \mathrm{~h}$. Control eyes were treated with PBS. Eyes were examined for various phenotypes at $24 \mathrm{~h}$. In 
some experiments, eyes were pretreated with antibiotic solution or PBS using the same timing prior to the above treatment.

Contact lens fitting. In some experiments, mice were fitted with custom-manufactured silicone hydrogel contact lens as previously described ${ }^{4}$. Contact lenses were fitted only onto the right eye and the contralateral eye used as a non-lens wearing control. Before fitting, contact lenses were removed from their packaging solution and soaked for $1 \mathrm{~h}$ in sterile phosphate buffered saline (PBS). After lens insertion under isoflurane anesthesia, mice were single-housed without enrichment to prevent lens removal. Pure-o'Cel paper bedding (The Andersons Inc., Maumee $\mathrm{OH}$ ) was used to reduce dust level. For overnight lens wear mice were returned to the animal care facility. Mice were monitored daily for lens retention, and evidence of pathology, e.g. discharge or corneal opacity. Any mouse that lost its contact lens was excluded from further experimentation.

Fluorescein in-situ hybridization (FISH). FISH-labeling was performed as previously described using universal 16S rRNA-targeted gene [Alexa488]-GCTGCCTCCCGTAGGAGT-[Alexa488] (Eurofins Genomics) ${ }^{8}$. Contact lenses or enucleated eyes were fixed in paraformaldehyde $(2 \%)$ for $24 \mathrm{~h}$ then stored in PBS at $4^{\circ} \mathrm{C}$ until processed for FISH. Samples were washed in $80 \% \mathrm{EtOH}, 95 \% \mathrm{EtOH}$ then PBS for 10 min each with rotation, placed in hybridization buffer [ $\mathrm{NaCl}(0.9 \mathrm{M})$, Tris- $\mathrm{HCl}(20 \mathrm{mM}, \mathrm{pH} 7.2)$ and SDS (0.01\%)] for incubation $\left(55^{\circ} \mathrm{C}, 30 \mathrm{~min}\right)$, then incubated overnight at $55^{\circ} \mathrm{C}$ with the $16 \mathrm{~S}$ rRNA probe $(100 \mathrm{nM})$. Samples were transferred to wash buffer solution $[\mathrm{NaCl}(0.9 \mathrm{M})$ and Tris- $\mathrm{HCl}(20 \mathrm{mM}, \mathrm{PH} 7.2)]$, then washed three times for 10 min each with rotation. For imaging, contact lenses were flat-mounted with Prolong Gold ${ }^{\text {TM }}$ and eyes were whole-mounted in a $47 \mathrm{~mm}$ Petri dish filled with PBS to cover the eyeball completely. Bacteria were imaged and quantified using ImageJ with maximum intensity projections.

AlkDala (Alkyne-Functionalized D-Alanine) Labeling. Viable bacteria on the murine ocular surface were also labeled using an alkyne functionalized D-alanine (alkDala) biorthogonal probe as previously described ${ }^{8,29,30}$. Freshly enucleated eyes were incubated in a solution of alkDala (10 mM) in Dulbecco's Modified Eagle Medium (DMEM) at 37॰ C for $2 \mathrm{~h}$ then transferred to pre-chilled $70 \% \mathrm{EtOH}$ then fixed for 20 min at $-20 \circ C$. After a thorough rinse in PBS, eyes were permeabilized in PBS with Triton-X100 (0.5\%) for 10 min with shaking at room temperature and washed 3 times for 5 min each in PBS with Triton-X100 $(0.1 \%)$ and $\mathrm{BSA}(3 \%)$ with shaking. Eyes were transferred to click-labeling cocktail [in PBS, TBTA (100 $\mu \mathrm{M}), \mathrm{CuSO}_{4}(1 \mathrm{mM})$, sodium ascorbate $(2 \mathrm{mM})$, a $488 \mathrm{~nm}$ azide-fluorophore $(10 \mu \mathrm{M})$, and BSA $(0.1$ $\mathrm{mg} / \mathrm{mL}$ )] for $1 \mathrm{~h}$ with shaking at room temperature. Eyes were imaged as described below.

Imaging. Confocal imaging of the mouse cornea (whole eyes ex vivo), or worn contact lenses, was performed using a 20x/1.00 NA water-dipping objective with an upright Olympus Fluoview FV1000 microscope. Whole mounted eyes were imaged using a $512 \mathrm{~nm}$ laser for detection of CD11c+-YFP cells (Yellow Fluorescence Protein) and a $559 \mathrm{~nm}$ laser for detecting red fluorescent cell membranes. A $488 \mathrm{~nm}$ laser was used to detect bacteria labeled with FISH or alkDala. For Z stacks at $1.00 \mu \mathrm{m}$ steps, images were collected from 4 or more random fields per sample. 3-D image reconstruction (reducing a 3-D image into 2-D) was achieved by projecting the maximum intensity of each pixel in a specific channel to the $z$ 
plane. Image-J (MorpholibJ tools collection) and Imaris (Bitplane) were used for image processing and analysis. For circularity measurements, morphological image analysis was performed using 3D segmentation in ImageJ and parameters related to z-projections used (circularity) to exclude artifacts due to lower $\mathrm{z}$ resolution.

Bacteria. Three bacterial species were used in this study. Corynebacterium mastiditis ${ }^{11}$ (kindly provided by Dr. Anthony St. Leger, University of Pittsburgh, PA), a clinical isolate of coagulase-negative Staphylococcus spp. and Pseudomonas aeruginosa strain PA01F. Bacteria were grown on tryptic soy agar plates at $37^{\circ} \mathrm{C}$ for $\sim 16 \mathrm{~h}$. A bacterial inoculum was prepared by suspension in PBS to a concentration of $\sim 10^{11} \mathrm{CFU} / \mathrm{ml}$ that was confirmed by viable counts. To inoculate mouse eyes, mice were anesthetized using ketamine $(80-100 \mathrm{mg} / \mathrm{Kg})$ and dexmedetomidine $(0.25-0.5 \mathrm{mg} / \mathrm{Kg})$ and the cornea of one eye was inoculated with $5 \mu \mathrm{l}$ of bacterial suspension. An additional $5 \mu \mathrm{l}$ of bacterial suspension was re-inoculated onto the corneas each hour up to $4 \mathrm{~h}$ ( 4 inoculations per eye in total). Mice remained anesthetized and covered under a heat lamp for the entire $4 \mathrm{~h}$ period. Subsequently, mice were subject to euthanasia and eyes examined by quantitative imaging.

\section{Experimental procedures.}

Procedure 1. Mice were divided into experimental groups containing 3-4 mice. One group was treated with antibiotic solution (cocktail), another with gentamicin alone, in one eye as described above. Contralateral eyes were treated with PBS as a control. After $24 \mathrm{~h}$, eyes were imaged ex vivo to measure corneal CD11c + cell numbers or subject to FISH labeling to determine if viable bacteria were present.

Procedure 2. Two groups of 3-4 mice were used. One group was treated with gentamicin solution (timing as above) in both eyes, the other group similarly treated in both eyes with PBS as a control. For the antibiotic treated group, after the first application of antibiotic, contact lens fitting was performed on one eye followed by remaining antibiotic applications. Contralateral eyes served as non-lens wearing antibiotic-treated controls. The PBS control group was treated similarly. One eye fitted with a contact lens followed by remaining PBS applications, contralateral eyes served as non-lens wearing PBS-treated controls.

Procedure 3. Similar to Procedure 2 with two groups of 3-4 mice: antibiotic treated group versus PBS control group. However, prior to each experiment, each group was pretreated with either antibiotic or PBS with the same timing as used for the primary experiment. In some experiments, however, antibiotic pretreatment was followed by contact lens application with PBS or PBS alone.

Statistical analysis. Data analysis was performed using Prism 9.0 for Mac, Microsoft Excel 2010, and the Statistical Package for Social Science for Mac version 27.0 (SPSS, Inc, Chicago, IL). The distribution of data was assessed by the normality test (Shapiro-Wilk test and Kolmogorov-Smirnov test), and since most data was normally distributed, it was expressed as the mean with standard deviation. One-way or Two-way ANOVA with Tukey's multiple comparisons test for post-hoc analysis. P values less than 0.05 were considered significant. 


\section{Declarations}

\section{Data availability}

All data generated or analyzed in this study are included in this published article and its Supplementary Information files.

\section{Acknowledgements}

This work was supported by the National Institutes of Health EY030350 (SMJF).

\section{Author contributions}

$A D, J L$, and TT performed the experiments.AD, DE and SF analyzed the data. AD, DE and SF wrote the manuscript. DE and SF designed the study and supervised the project.

\section{Competing financial interests}

The authors have no conflicts of interest to disclose in relation to the work presented.

\section{References}

1. Fleiszig, S. M. J. et al. Contact lens-related corneal infection: Intrinsic resistance and its compromise. Prog. Retin. Eye Res. 100804 (2019) doi:10.1016/J.PRETEYERES.2019.100804.

2. Hamrah, P. \& Dana, M. R. Corneal antigen-presenting cells. Chem. Immunol. Allergy (2007) doi:10.2147/OTT.S85383.

3. Chinnery, H. R. \& Hons, B. S. Dendritic cells and macrophages in the mammalian cornea: distribution , morphology, phenotype and By. repository.uwa.edu.au (2008).

4. Metruccio, M. M. E. et al. A novel murine model for contact lens wear reveals clandestine IL-1R dependent corneal parainflammation and susceptibility to microbial keratitis upon inoculation with Pseudomonas aeruginosa. Ocul. Surf.17, 119-133 (2019).

5. Alzahrani, Y., Pritchard, N. \& Efron, N. Changes in corneal Langerhans cell density during the first few hours of contact lens wear. Contact Lens Anterior Eye39, 307-310 (2016).

6. Efron, N. Contact lens wear is intrinsically inflammatory. Clin. Exp. Optom.100, 3-19 (2017).

7. Metruccio, M. M. E. et al. Contributions of MyD88-dependent receptors and CD11c-positive cells to corneal epithelial barrier function against Pseudomonas aeruginosa. Sci. Rep.7, 13829 (2017).

8. Wan, S. J. et al. IL-1R and MyD88 Contribute to the Absence of a Bacterial Microbiome on the Healthy Murine Cornea. Front. Microbiol.9, 1117 (2018).

9. Willcox, M. D. P. Characterization of the normal microbiota of the ocular surface. Experimental Eye Research vol. 117 99-105 (2013). 
10. Ozkan, J., Coroneo, M., Willcox, M., Wemheuer, B. \& Thomas, T. Identification and Visualization of a Distinct Microbiome in Ocular Surface Conjunctival Tissue. Investig. Opthalmology Vis. Sci.59, 4268 (2018).

11. St Leger, A. J. et al. An ocular commensal protects against corneal infection by driving an Interleukin 17 response from mucosal $ү \delta$ T cells HHS Public Access. Immunity47, 148-158 (2017).

12. Fleiszig, S. M. J. \& Efron, N. Microbial flora in eyes of current and former contact lens wearers. J. Clin. Microbiol.30, 1156-1161 (1992).

13. Fleiszig, S. M., Efron, N. \& Pier, G. B. Extended contact lens wear enhances Pseudomonas aeruginosa adherence to human corneal epithelium. Invest Ophthalmol Vis Sci33, 2908-2916 (1992).

14. Willcox, M. D. P. Microbial adhesion to silicone hydrogel lenses: A review. in Eye and Contact Lens vol. 39 61-66 (2013).

15. Fletcher, E. L., Fleiszig, S. M. \& Brennan, N. A. Lipopolysaccharide in adherence of Pseudomonas aeruginosa to the cornea and contact lenses. Invest Ophthalmol Vis Sci34, 1930-1936 (1993).

16. Willcox, M. D., Harmis, N. Y. \& Holden, B. A. Bacterial populations on high-Dk silicone hydrogel contact lenses: effect of length of wear in asymptomatic patients. Clin Exp.Optom (2002).

17. Wan, S. J., Ma, S., Evans, D. J. \& Fleiszig, S. M. J. Resistance of the murine cornea to bacterial colonization during experimental dry eye. PLoS One15, (2020).

18. Efron, N. Contact lens wear is intrinsically inflammatory. Clin. Exp. Optom.100, 3-19 (2017).

19. Efron, N. Contact lens-induced changes in the anterior eye as observed in vivo with the confocal microscope. Prog. Retin. Eye Res.26, 398-436 (2007).

20. Krause, K. M., Serio, A. W., Kane, T. R. \& Connolly, L. E. Aminoglycosides: An Overview. Cold Spring Harb. Perspect. Med.6, (2016).

21. St. Leger, A. J. et al. An Ocular Commensal Protects against Corneal Infection by Driving an Interleukin-17 Response from Mucosal yठ T Cells. Immunity47, 148-158.e5 (2017).

22. Gauguet, S. et al. Intestinal microbiota of mice influences resistance to Staphylococcus aureus pneumonia. Infect. Immun. (2015) doi:10.1128/IAI.00037-15.

23. Kugadas, A., Wright, Q., Geddes-McAlister, J. \& Gadjeva, M. Role of microbiota in strengthening ocular mucosal barrier function through secretory IgA. Investig. Ophthalmol. Vis. Sci. (2017) doi:10.1167/iovs.17-22119.

24. Kugadas, A. et al. Impact of Microbiota on Resistance to Ocular Pseudomonas aeruginosa-Induced Keratitis. PLOS Pathog.12, e1005855 (2016).

25. Yang, J. H. et al. Antibiotic-Induced Changes to the Host Metabolic Environment Inhibit Drug Efficacy and Alter Immune Function. Cell Host Microbe22, 757-765.e3 (2017).

26. Prokhorova, I. et al. Aminoglycoside interactions and impacts on the eukaryotic ribosome. Proc. Natl. Acad. Sci. U. S. A.114, E10899-E10908 (2017).

27. Allam, R. et al. Cutting edge: cyclic polypeptide and aminoglycoside antibiotics trigger IL-1 $\beta$ secretion by activating the NLRP3 inflammasome. J. Immunol.186, 2714-8 (2011). 
28. Kagi, T. et al. The polypeptide antibiotic polymyxin B acts as a pro-inflammatory irritant by preferentially targeting macrophages. J. Antibiot. (Tokyo).75, 29-39 (2022).

29. Shieh, P., Siegrist, M. S., Cullen, A. J. \& Bertozzi, C. R. Imaging bacterial peptidoglycan with nearinfrared fluorogenic azide probes. Proc. Natl. Acad. Sci. U. S. A. (2014) doi:10.1073/pnas.1322727111.

30. Siegrist, M. S., Swarts, B. M., Fox, D. M., Lim, S. A. \& Bertozzi, C. R. Illumination of growth, division and secretion by metabolic labeling of the bacterial cell surface. FEMS Microbiology Reviews vol. 39 184-202 (2015).

\section{Figures}

A

PBS



Antibiotic cocktail

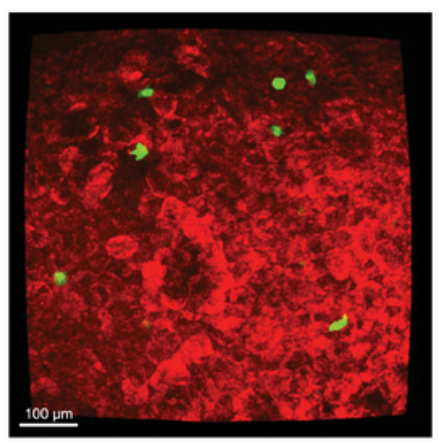

B

Gentamicin



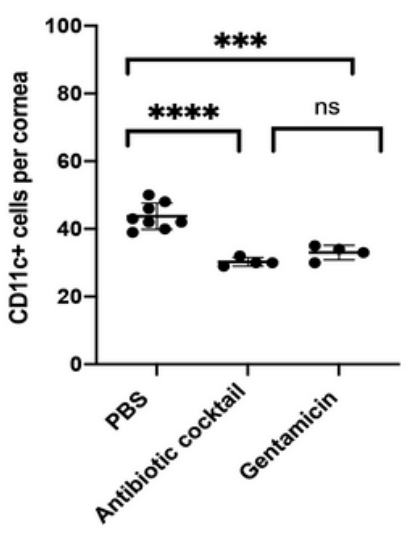

Figure 1

Antibiotic-treated corneas of CD11c-YFP mice show a decreasein baseline CD11c+ cells. (a) Maximum intensity z-projection of the YFP signal from CD11c+ cells (yellow) in the central cornea of healthy mouse eyes treated with antibiotic cocktail orgentamicin(see Methods) show reduced numbers of CD11c+ cells versus contralateral PBS treated controls at $24 \mathrm{~h}$. Scale bar $=100 \mu \mathrm{m}$. (b) Quantification of CD11c+ cells

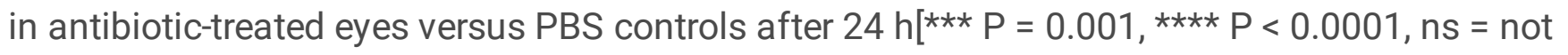
significant; One-way ANOVA with Tukey's multiple comparisons test]. 


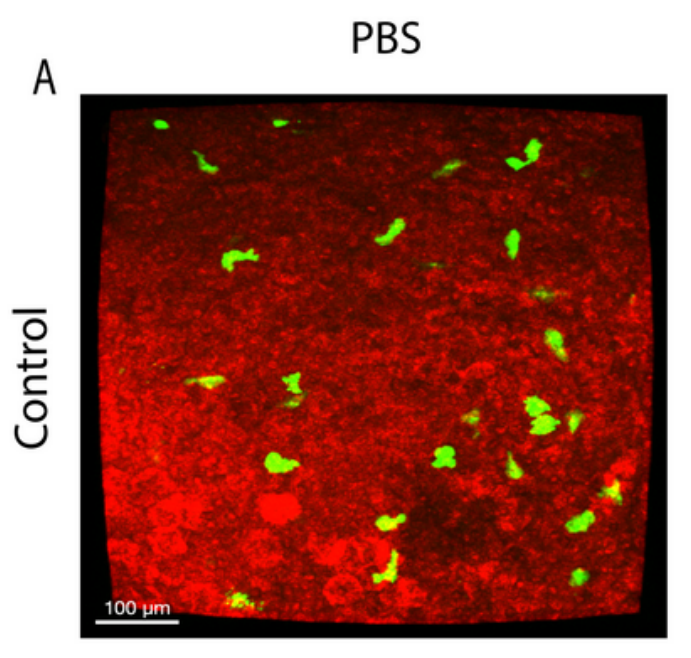

\section{Gentamicin}
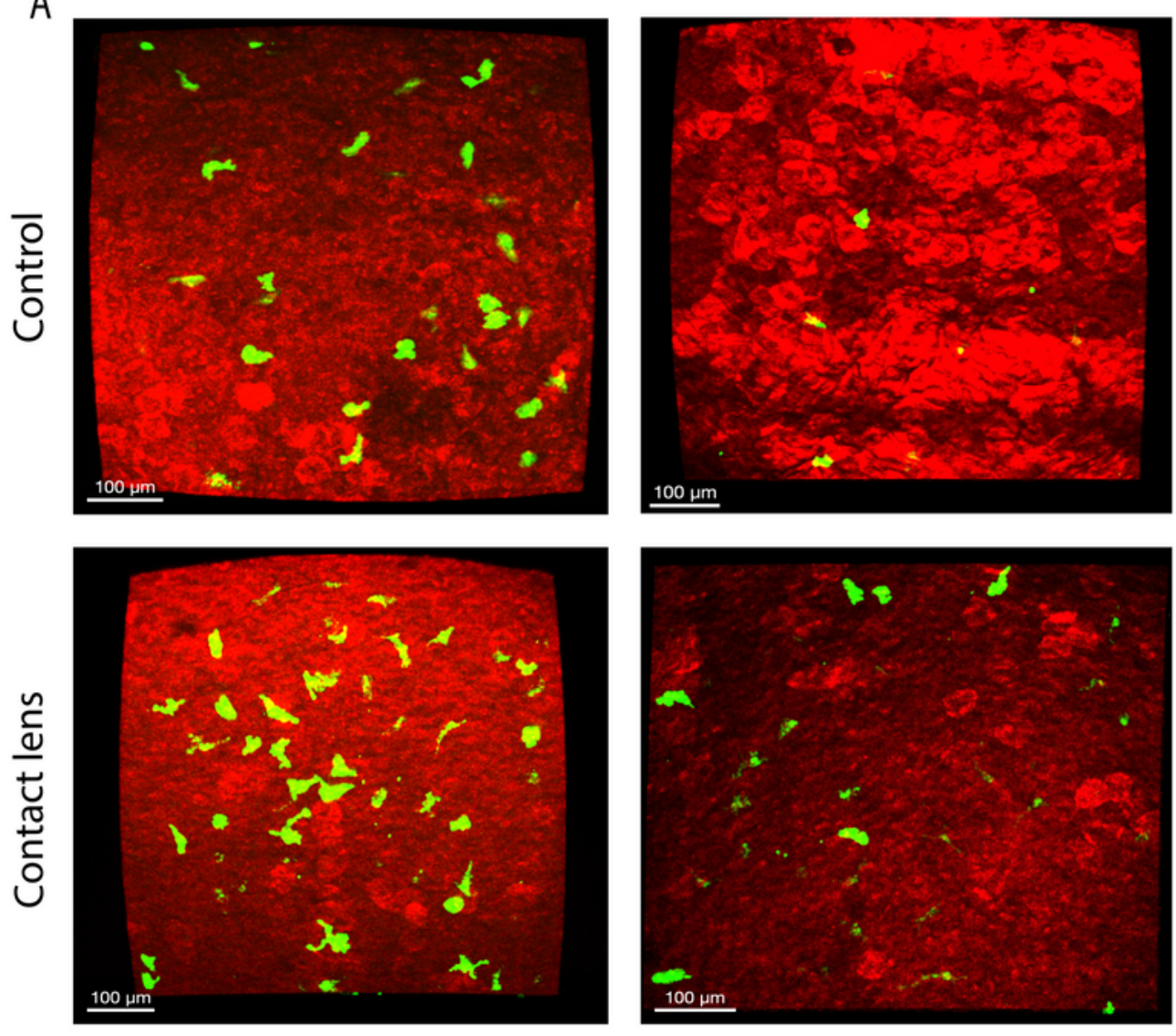

B

C
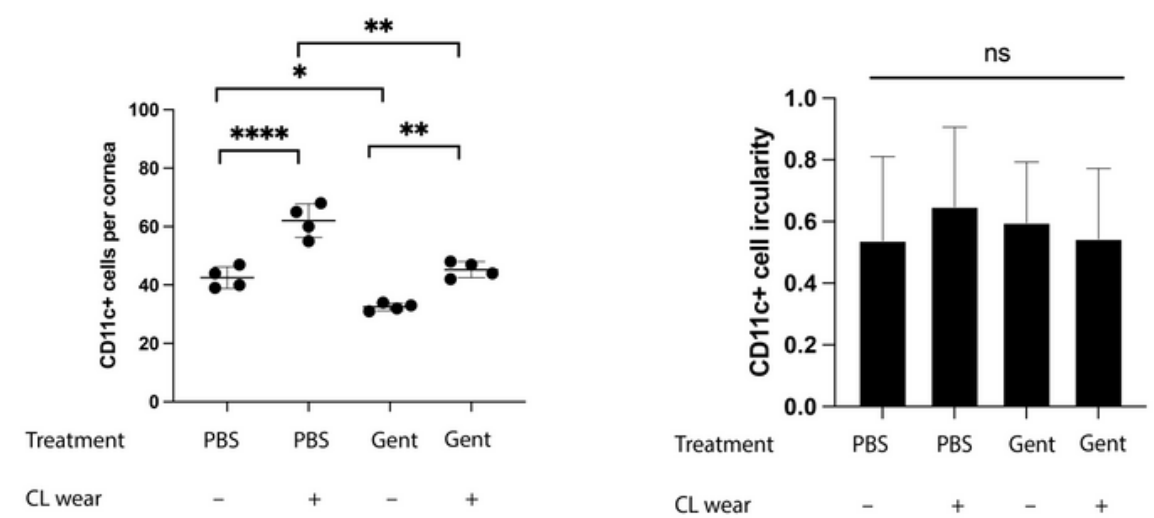

\section{Figure 2}

Gentamicin treatment did not prevent the $24 \mathrm{~h}$ CD11c+ cell response to contact lens wear. (a) Maximum intensity z-projection of the YFP signal from CD11 c+ cells in the centralcornea after $24 \mathrm{~h}$ of contact lens $(\mathrm{CL})$ wear versus non-lens wearing contralateral controls with PBS or gentamicin treatment. (b) Quantification of the CD11 + cell responses after $24 \mathrm{~h}$. Lens-associated CD11c+ cell responses were evident in each instance. Scale bar $=100 \mu \mathrm{m} .\left[{ }^{*} \mathrm{P}<0.05,{ }^{*} \mathrm{P}=0.002, * \star \star \star \mathrm{P}<0.0001\right.$; One-way ANOVA 
with Tukey's multiple comparisons test]. (c) Quantification of morphological parameters (circularity) of $\mathrm{CD} 11 \mathrm{c}+$ cells from the same experiment. There was no significant difference between groups [ns = not significant; One-way ANOVA with Tukey's multiple comparisons test].
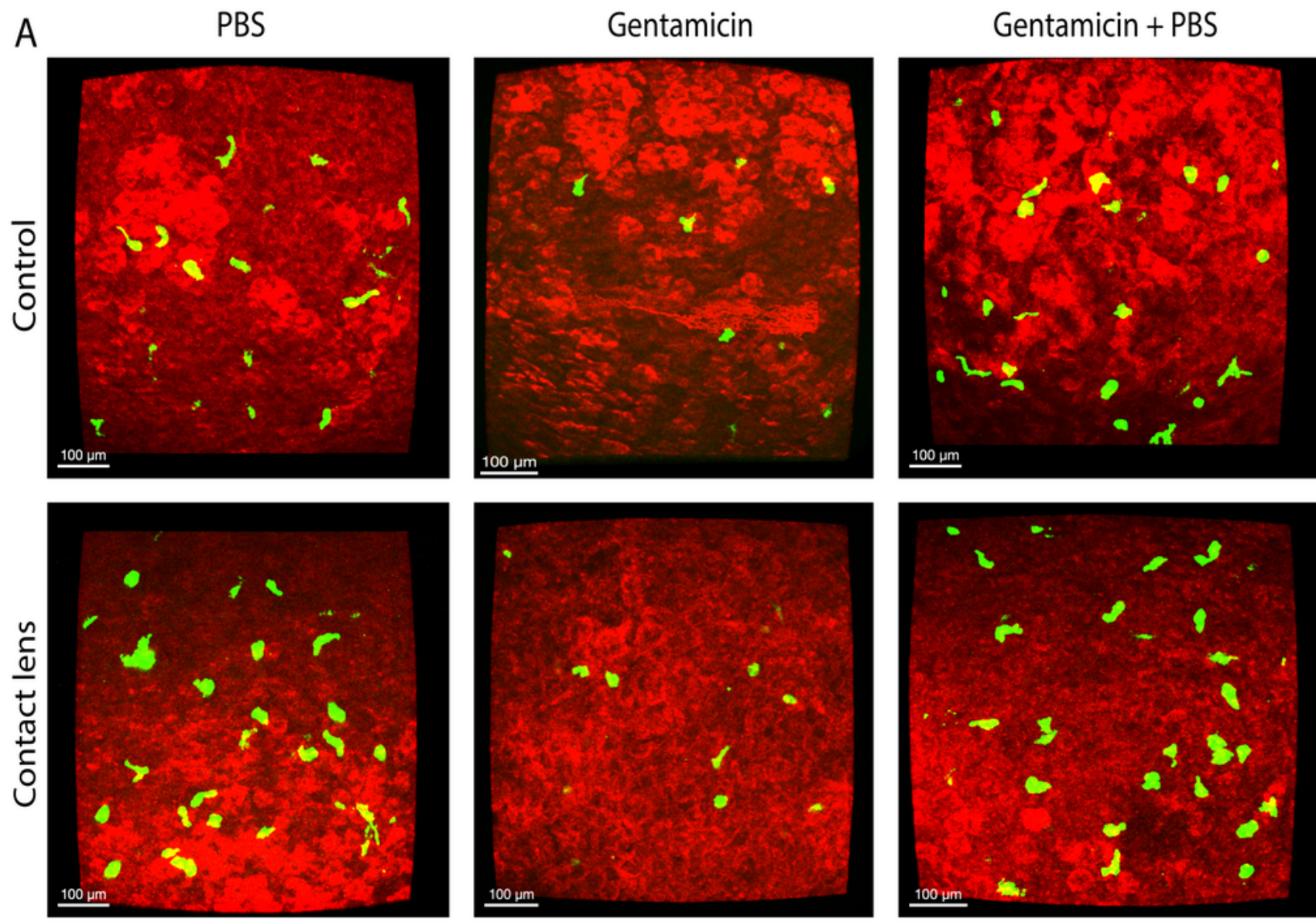

B

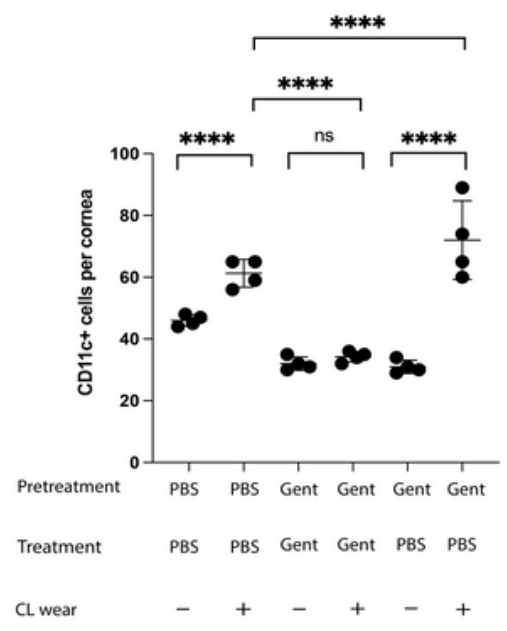



C
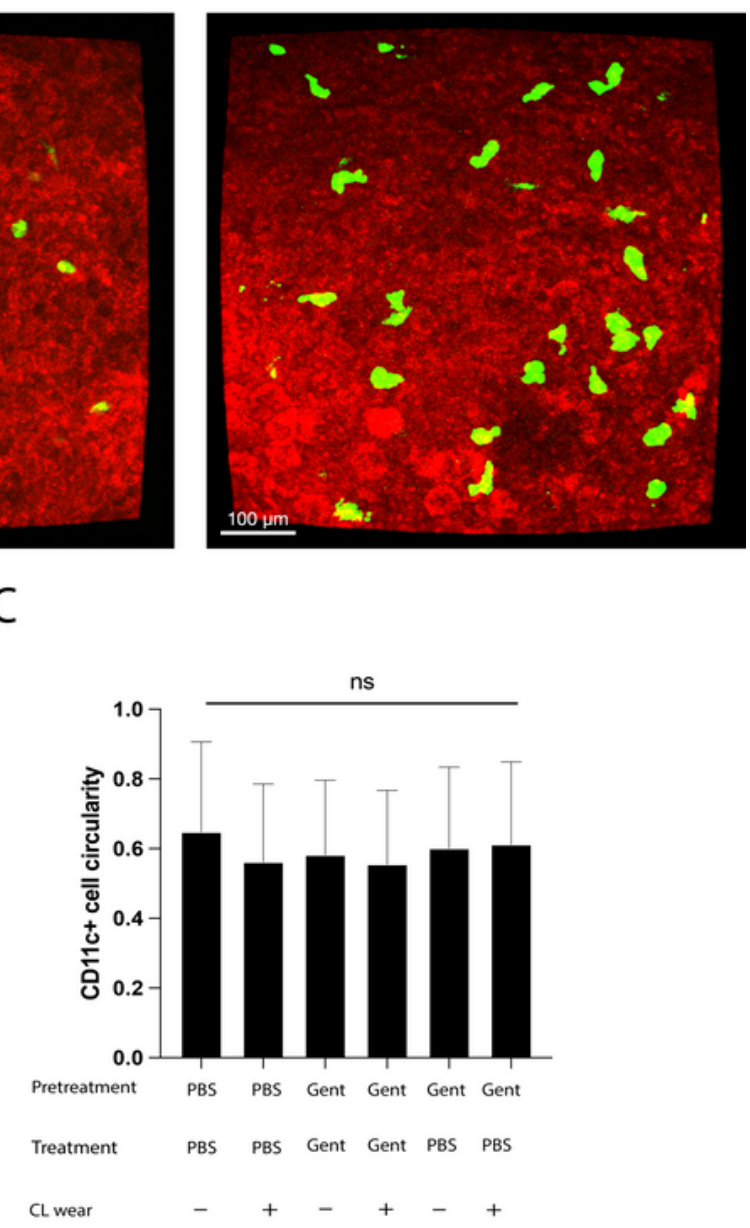

Figure 3 
Contact lens-associated $\mathrm{CD} 11 \mathrm{c}+$ cell responses at $24 \mathrm{~h}$ are preventedby gentamicin pretreatment combined with continued gentamicin during lens wear. (a) Maximum intensity z-projection of the YFP signal from CD11c+ cells in the central cornea under different conditions of PBS or gentamicin pretreatment and treatment with or without contact lens $(C L)$ wear. Lens-associated CD11c+ responses were prevented only if eyes were gentamicin pretreated combined with treatment during $C L$ wear. Scale

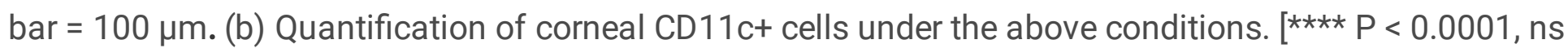
= not significant; One-way ANOVA with Tukey's multiple comparisons test]. (c) Quantification of morphological parameters (circularity) of CD11c+ cells from the same experiment. There was no significant difference between groups [ns = not significant; One-way ANOVA with Tukey's multiple comparisons test]. 


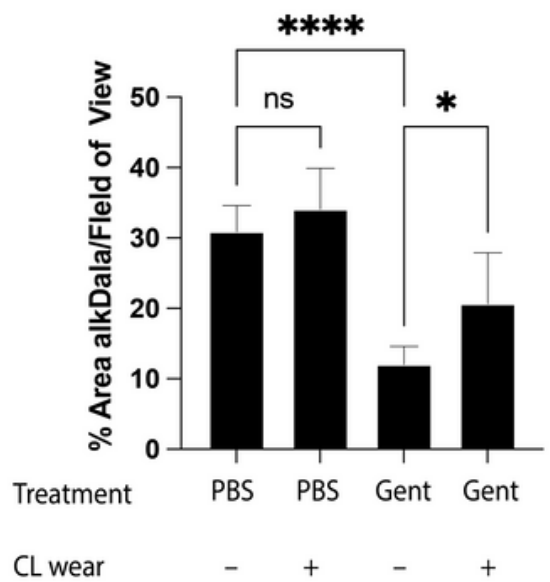

B

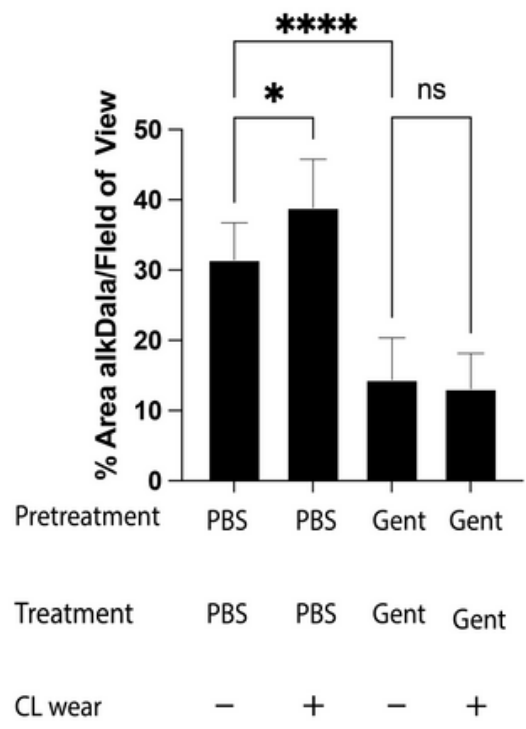

Figure 4

AlkDala labeling of viable conjunctival-associated bacteria. Quantification of alkDala(bacterial) fluorescence on theconjunctiva with(a) PBS or gentamicin treatment with contact lens (CL) wear for $24 \mathrm{~h}$ or (b) PBS or gentamicin pretreatment followed by the same treatments with or without CL wear. Gentamicin treatment/pretreatment was associated with decreased bacterial fluorescence on the conjunctiva with or without CL wear and these changes correlated with corneal CD11c+ changes under 
respective conditions [ ${ }^{\star} \mathrm{P}<0.05,{ }^{\star \star \star \star} \mathrm{P}<0.0001, \mathrm{~ns}=$ not significant; Two-way ANOVA with Tukey's multiple comparisons test].
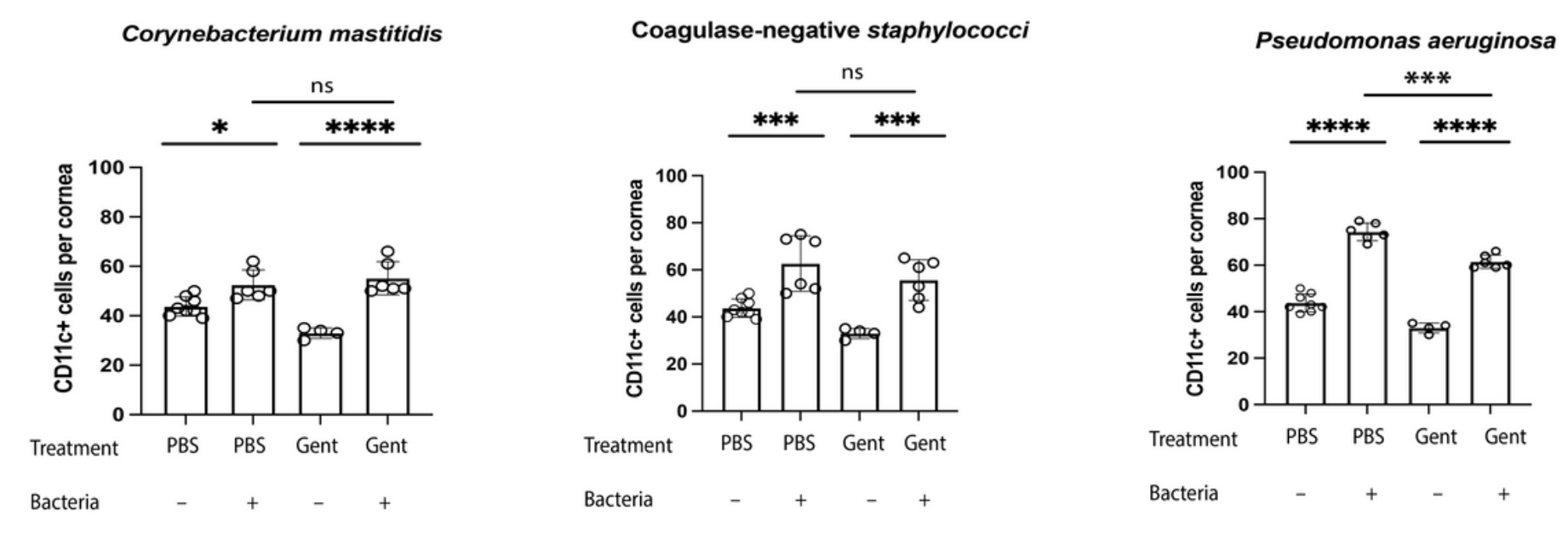

\section{Figure 5}

Quantification of corneal CD11c+ cells after inoculation with (a) Corynebacterium mastitidis (b) Coagulase-negativeStaphylococcior (c) Pseudomonas aeruginosa $\left(\sim 10^{11} \mathrm{CFU} / \mathrm{ml}\right)$ for 4 hbeginning at 24 $\mathrm{h}$ after PBS or gentamicin treatment.Addition of Gram-positive commensal bacteria $(\mathrm{a}, \mathrm{b})$ increased CD11c+ numbers in antibiotic-treated corneas to levels similar tocontrols $[* \mathrm{P}<0.05$, $* \star \star P<0.001$, $0.0001, \mathrm{~ns}=$ not significant; One-way ANOVA with Tukey's multiple comparisons test].

\section{Supplementary Files}

This is a list of supplementary files associated with this preprint. Click to download.

- NewSupplementalFigureS1.tif

- NewSupplementalFigures2.tif 